Article

\title{
Relations between Clifford Algebra and Dirac Matrices in the Presence of Families
}

\author{
Dragan Lukman ${ }^{1}$, Mickael Komendyak ${ }^{2}$ and Norma Susana Mankoč Borštnik ${ }^{3, *}$ \\ CAMTP, University of Maribor, 2000 Maribor, Slovenia; dragan.lukman@guest.arnes.si \\ 2 Department of Physics, University of Warwick, Coventry CV4 7AL, UK; komendyak@gmail.com \\ 3 Department of Physics, University of Ljubljana, 1000 Ljubljana, Slovenia \\ * Correspondence: norma.mankoc@fmf.uni-lj.si
}

Received: 31 March 2020; Accepted: 23 June 2020; Published: 29 June 2020

\begin{abstract}
The internal degrees of freedom of fermions are in the spin-charge-family theory described by the Clifford algebra objects, which are superposition of an odd number of $\gamma^{a \prime}$. Arranged into irreducible representations of "eigenvectors" of the Cartan subalgebra of the Lorentz algebra $S^{a b}\left(=\left.\frac{i}{2} \gamma^{a} \gamma^{b}\right|_{a \neq b}\right)$ these objects form $2^{\frac{d}{2}-1}$ families with $2^{\frac{d}{2}-1}$ family members each. Family members of each family offer the description of all the observed quarks and leptons and antiquarks and antileptons, appearing in families. Families are reachable by $\tilde{S}^{a b}=\left.\frac{1}{2} \tilde{\gamma}^{a} \tilde{\gamma}^{b}\right|_{a \neq b}$. Creation operators, carrying the family member and family quantum numbers form the basis vectors. The action of the operators $\gamma^{a}$ 's, $S^{a b}, \tilde{\gamma}^{a}$ 's and $\tilde{S}^{a b}$, applying on the basis vectors, manifests as matrices. In this paper the basis vectors in $d=(3+1)$ Clifford space are discussed, chosen in a way that the matrix representations of $\gamma^{a}$ and of $S^{a b}$ coincide for each family quantum number, determined by $\tilde{S}^{a b}$, with the Dirac matrices. The appearance of charges in Clifford space is discussed by embedding $d=(3+1)$ space into $d=(5+1)$-dimensional space. The achievements and predictions of the spin-charge-family theory is also shortly presented.
\end{abstract}

Keywords: dirac matrices; clifford algebra; Kaluza-Klein theories; higher dimensional spaces; beyond the standard model; lepton and quark families

PACS: 04.50.-h; 04.50.Cd; 11.30.Ly

\section{Introduction}

Motivation: More than 50 years ago the electroweak (and colour) standard model offered an elegant new step in understanding the origin of elementary fermion and boson fields by postulating

i. the existence of massless family members with the charges in fundamental representations of the groups-the colour triplet quarks and colourless leptons the left handed members as the weak charged doublets, the right handed weak chargeless members, the left handed quarks distinguishing in the hyper charge from the left handed leptons, each right handed member having a different hyper charge, the existence of the corresponding anti fermions-the existence of massless families to each family member,

ii. the existence of massless vector gauge fields to the observed charges of the family members, carrying charges in the adjoint representations of the charge groups,

iii. the existence of the massive scalar fields with the nonzero vacuum expectation value carrying the weak charge $\pm \frac{1}{2}$ and the hyper charge $\mp \frac{1}{2}$ (like if it would be in the fundamental representation of the 
weak group), determining the masses of quarks and leptons and of the weak bosons, the existence of the Yukawa couplings determining the properties of fermions.

The spin-charge-family theory [1-6], describing the internal degrees of freedom with the odd Clifford algebra in $d \geq(13+1)$, explains all the postulated properties of quarks and leptons and antiquarks and antileptons [[2-4] and the references therein].

Statement 1: One irreducible representation of the Lorentz group in the internal space of fermions described by odd Clifford algebra contains, if analyzed with respect to the standard model groups $S O(3,1), S U(3), S U(2), U(1)$, all the quarks and antiquarks, all the leptons and antileptons, with the quantum numbers postulated by the standard model [[3,4] and the references there in].

Statement 2: The odd Clifford algebra in $d \geq(13+1)$ offers-after the break of the starting symmetry-two decoupled groups of four families: The fourth family to the observed three families of quarks and leptons is predicted to have the mass of quarks at around $1 \mathrm{TeV}$ or larger, the lightest neutron of the upper four families of quarks and leptons is the candidate, together with their lightest neutrino, to form the dark matter $[7,8]$.

The fact that the odd Clifford algebra offers the description of quarks and leptons and antiquarks and antileptons as assumed by the standard model suggests that the standard model vector gauge fields-gravitational, colour, weak and hyper- have the common origin.

Statement 3: The vielbeins and the two kinds of the spin connection fields in $d=(13+1)$ manifest in $d=(3+1)$ all the known vector gauge fields postulated by the standard model as well a several scalar fields, those carrying the weak and hyper charge equal to $\left( \pm \frac{1}{2}, \mp \frac{1}{2}\right)$, respectively [2,3,9]) offering the explanation for the Higgs and the Yukawa couplings, as well as those causing in the expanding universe the matter-antimatter asymmetry [2].

The achievements and predictions of the spin-charge-family theory, to which the above statements 1,2,3 refer, are shortly presented in Appendix A.

We demonstrate in this paper the appearance of families, when internal degrees of freedom are described with the odd Clifford algebra in $d=(3+1)$, and the appearance of charges and families, when internal degrees of freedom are described with the odd Clifford algebra in $d=(5+1)$, we present the basis vectors of each irreducible representation of each family in both cases as well as the corresponding matrix representations of the operators of the Clifford algebra objects. We make the choice of the basis vectors in the way that the matrix elements coincide for each family with the Dirac ones up to a phase.

\subsection{Mathematical Background}

In the Grassmann graded algebra of anticommuting coordinates $\theta^{a}$ there are in $d$-dimensional space $2^{d}$ vectors, which define, together with the corresponding derivatives $\frac{\partial}{\partial \theta_{a}}$, two kinds of the Clifford algebra objects: $\gamma^{a}$ and $\tilde{\gamma}^{a}[1,5,6,10]$, both with the anticommutation properties of the Dirac $\gamma^{a}$ matrices, while the anticommutators among $\gamma^{a}$ and $\tilde{\gamma}^{b}$ are equal to zero.

$$
\begin{aligned}
\left\{\gamma^{a}, \gamma^{b}\right\}_{+} & =2 \eta^{a b}=\left\{\tilde{\gamma}^{a}, \tilde{\gamma}^{b}\right\}_{+}, \quad\left\{\gamma^{a}, \tilde{\gamma}^{b}\right\}_{+}=0, \\
\left(\gamma^{a}\right)^{+} & =\eta^{a a} \gamma^{a}, \quad\left(\tilde{\gamma}^{a}\right)^{+}=\eta^{a a} \tilde{\gamma}^{a}, \\
S^{a b} & =\frac{i}{4}\left(\gamma^{a} \gamma^{b}-\gamma^{b} \gamma^{a}\right), \quad \tilde{S}^{a b}=\frac{i}{4}\left(\tilde{\gamma}^{a} \tilde{\gamma}^{b}-\tilde{\gamma}^{b} \tilde{\gamma}^{a}\right), \\
\left\{S^{a b}, \tilde{S}^{a b}\right\}_{+} & =0 \\
(a, b) & =(0,1,2,3,5, \cdots, d) .
\end{aligned}
$$

The two Clifford algebras, $\gamma^{a \prime}$ s and $\tilde{\gamma}^{a \prime}$, are obviously completely independent and form two independent spaces, each with $2^{d}$ vectors [11]. 
Sacrificing the space of $\tilde{\gamma}^{a}$ 's by defining

$$
\tilde{\gamma}^{a} B\left(\gamma^{a}\right)=(-)^{B} i B \gamma^{a},
$$

with $(-)^{B}=-1$, if $B$ is an odd product of $\gamma^{a}$ 's, otherwise $(-)^{B}=1$ [6], we end up with vector space of $2^{d}$ degrees of freedom, defined by $\gamma^{a}$ s only. The reader can easily prove that the relation of Equation (2) keeps anticommutation and commutation relations in Equation (1) unchanged.

A general vector can correspondingly be written as

$$
\mathbf{B}=\sum_{k=0}^{d} a_{a_{1} a_{2} \ldots a_{k}} \gamma^{a_{1}} \gamma^{a_{2}} \ldots \gamma^{a_{k}} \mid \psi_{0}>, \quad a_{i} \leq a_{i+1},
$$

where $\mid \psi_{0}>$ is the vacuum state.

We arrange these vectors as products of nilpotents and projectors

$$
\begin{array}{ll}
\stackrel{a b}{(k)}:=\frac{1}{2}\left(\gamma^{a}+\frac{\eta^{a a}}{i k} \gamma^{b}\right), & (\stackrel{a b}{(k)})^{2}=0 . \\
\stackrel{a b}{[k}:=\frac{1}{2}\left(1+\frac{i}{k} \gamma^{a} \gamma^{b}\right), & ([k])^{2}=[k],
\end{array}
$$

where $k^{2}=\eta^{a a} \eta^{b b}$. Their Hermitian conjugated values follow from Equation (1).

$$
\stackrel{a b}{+}(k)=\eta^{a a}(\stackrel{a b}{(-k),}, \quad[k]=[k] .
$$

After choosing the Cartan subalgebra of the Lorentz algebra $S^{a b}$ in the internal space of $\gamma^{a \prime}$ s and making the equivalent choice also for $\tilde{S}^{a b}$

$$
\begin{aligned}
& S^{03}, S^{12}, S^{56}, \cdots, S^{d-1 d}, \\
& \tilde{S}^{03}, \tilde{S}^{12}, \tilde{S}^{56}, \cdots, \tilde{S}^{d-1 d},
\end{aligned}
$$

we make the basis vectors in Clifford space to be the eigenstates of the Cartan subalgebra, Equation (6), with the eigenvalues $S^{a b} \stackrel{a b}{(k)}=\frac{1}{2} k \stackrel{a b}{(k)}, S^{a b} \stackrel{a b}{[k]}=\frac{1}{2} k \stackrel{a b}{[k]}$. Since all the relations of Equation (1) remain unchanged after the assumption of Equation (2), it follows that each irreducible representation of the Lorentz algebra $S^{a b}$ receives the additional quantum number $f$, defined by $\tilde{S}^{a b}$ of Equation (6). It then follows

$$
\begin{aligned}
& S^{a b} \stackrel{a b}{(k)}=\frac{k}{2} \stackrel{a b}{(k)}, \quad \quad \tilde{S}^{a b} \stackrel{a b}{(k)}=\frac{k}{2} \stackrel{a b}{(k)},
\end{aligned}
$$

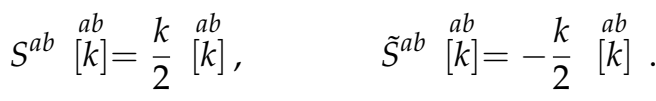

Equation (7) demonstrates that the eigenvalues of $S^{a b}$ on nilpotents and projectors-generated by $\gamma^{a \prime}$ s-differ from the eigenvalues of $\tilde{S}^{a b}$. Nilpotents are the superposition of odd number of $\gamma^{a \prime}$ s, projectors have an even Clifford character. 
States, which are products of projectors and nilpotents, have well defined handedness of both kinds, $\Gamma^{(d)}$ and $\tilde{\Gamma}^{(d)}$

$$
\begin{aligned}
& \Gamma^{(d)}:=(i)^{d / 2} \quad \prod_{a}\left(\sqrt{\eta^{a a}} \gamma^{a}\right), \text { if } \quad d=2 n, \\
& \tilde{\Gamma}^{(d)}:={ }_{(i)^{d / 2}} \prod_{a}\left(\sqrt{\eta^{a a}} \tilde{\gamma}^{a}\right), \text { if } \quad d=2 n .
\end{aligned}
$$

The spin-charge-family theory [1-6] of N.S. Mankoč Borštnik uses odd products of nilpotents, $\stackrel{a b}{k})=\frac{1}{2}\left(\gamma^{a}+\frac{\eta^{a a}}{i k} \gamma^{b}\right)$, and projectors, $[k]=\frac{a b}{2}\left(1+\frac{i}{k} \gamma^{a} \gamma^{b}\right)$, to define $2^{d-1}$ basis vectors in this space of the Clifford graded algebra [1-4].

These Clifford odd basis vectors split further into two groups, which are Hermitian conjugated to each other $[[10,11]$ and references therein]. The choice of the vacuum state (which is for the case that $d=(5+1)$ presented in Equation (9)) makes one of these two groups to have properties of creation operators. Their Hermitian conjugated partners have properties of annihilation operators.

The creation operators consist of $2^{\frac{d}{2}-1}$ irreducible representations with respect to $S^{a b}$, carrying the family quantum number determined by $\tilde{S}^{a b}$ 's belonging to Equation (6). Each family has $2^{\frac{d}{2}-1}$ members.

These creation and annihilation operators fulfill the anticommutation relations postulated by Dirac [12] for second quantized fermions, and consequently explain $[10,11]$ the Dirac's postulates.

In this spin-charge-family theory $S^{a b}$ determine in $d=(3+1)$ space, which is a part of $d=(13+1)$ dimensional space, spins, handedness and charges of quarks and leptons, while $\tilde{S}^{a b}$ determine families of quarks and leptons.

Let us repeat: Clifford space define all the internal degrees of freedom of fermions-spins, handedness, charges and families. Charges are expressed by the Cartan subalgebra of $S^{a b}$ when embedding $d=(3+1)$ space into $d \geq(5+1)$ space. Family quantum numbers are determined by $\tilde{S}^{a b}$.

In this paper we discuss the simple case with $d=(5+1)$.

In Section 2 the properties of products of nilpotents and projectors are presented, arranged into eigenvectors of the Cartan subalgebra, when $d=(3+1)$-dimensional space is embedded into $d=(5+1)$-dimensional space. Nilpotents and projectors define the internal vector space of fermions so that the spin in $d=(5,6)$ manifests as a charge of fermions in $d=(3+1)$.

In Section 2.3 the matrix representations of vectors are presented.

\section{Properties of Vectors in Clifford Space}

In References $[10,11]$ the fact that the Clifford vectors, spanned by products of an odd number of $\gamma^{a}$ 's, are fulfilling the anticommutation relations postulated by Dirac for the second quantized fermions, are discussed. Let us illustrate how this happens in the case that $d=(5+1)$.

Let us denote vectors in $d=(5+1)$ of an odd Clifford character (they are superposition of an odd products of $\gamma^{a \prime}$ ), presented in Table 1 , as products of nilpotents and projectors, by $\hat{b}_{m}^{f \dagger}$ (the third column on Table 1). The member quantum number $m=(c h, s)$ includes the charge $c h$ and the spin $s$, the charge concerns the eigenvalue of $S^{56}$, the spin the eigenvalue of $S^{12}$. The corresponding Hermitian conjugated partner (the fourth column on Table 1) is denoted by $\left(\hat{b}_{m}^{f \dagger}\right)^{\dagger}=\hat{b}_{m}^{f}$.

The first member $m=\left(\frac{1}{2}, \frac{1}{2}\right)$ of the first family $a$, which is the product of three nilpotents, is correspondingly denoted by $\hat{b}_{\left(\frac{1}{2}, \frac{1}{2}\right)}^{a+}:=\left(\begin{array}{c}03 \\ +\end{array}\right)\left(\begin{array}{l}12 \\ +\end{array}\right) \mid \stackrel{56}{(+)}$. All the other vectors $\hat{b}_{m}^{f \dagger}$ of the family $f=a$ follow by the application of $S^{a b}$. The families $\hat{b}_{m}^{f \dagger}, f=(b, c, d)$ follow from $f=a$ by the application of $\tilde{S}^{a b}$. The Hermitian conjugated partners follow by the application of Equation (1). 
Table 1, taken from Table IV of Reference [10], represents four families of Clifford odd vectors and their Hermitian conjugated partners. All the families have the same quantum numbers $m$ of the corresponding members $\left(S^{03}, S^{12}, S^{56}\right)$ each family carries its own family quantum number $f$.

Table 1. The basis creation operators $\hat{b}_{m}^{f+}$, which are sums of odd products of $\gamma^{a}$ 's, and their annihilation partners $\hat{b}_{m}^{f}$ are presented for the $d=(5+1)$-dimensional case. Here $m=(c h, s)$, ch represents the spin in $d=(5,6)$, manifesting in $d=(3+1)$ the charge, and $s$ represents the spin, that is the eigenvalue of $S^{12}$, according to the choice of the Cartan subalgebra, Equation (6). The basis creation operators are the products of nilpotents and projectors, which are the "eigenstates" of the Cartan subalgebra generators, $\left(S^{03}, S^{12}, S^{56}\right)$ and $\left(\tilde{S}^{03}, \tilde{S}^{12}, \tilde{S}^{56}\right)$, presented in Equation (6).

\begin{tabular}{|c|c|c|c|c|c|c|c|c|c|c|}
\hline$f$ (amily) $m$ & $(c h, s)$ & $\hat{b}_{m}^{f \dagger}$ & $\hat{\boldsymbol{b}}_{m}^{f}$ & $S^{03}$ & $S^{12}$ & $S^{56}$ & $\Gamma^{(3+1)}$ & $\tilde{S}^{03}$ & $\tilde{S}^{12}$ & $\tilde{S}^{56}$ \\
\hline$a 1$ & $\left(\frac{1}{2}, \frac{1}{2}\right)$ & $\begin{array}{ccc}03 & 12 & 56 \\
(+i) & (+) \mid(+)\end{array}$ & $(-) \stackrel{56}{(-)} \mid(-){ }^{12}(-)(-i)$ & $\frac{i}{2}$ & $\frac{1}{2}$ & $\frac{1}{2}$ & 1 & $\frac{i}{2}$ & $\frac{1}{2}$ & $\frac{1}{2}$ \\
\hline$a 2$ & $\left(\frac{1}{2},-\frac{1}{2}\right)$ & {$\left[\begin{array}{ll}03 & 12 \\
{[-i} & {[-]}\end{array} \mid(+)\right.$} & $(-){ }^{56}(-) \mid[-][-i]$ & $-\frac{i}{2}$ & $-\frac{1}{2}$ & $\frac{1}{2}$ & 1 & $\frac{i}{2}$ & $\frac{1}{2}$ & $\frac{1}{2}$ \\
\hline$a 3$ & $\left(-\frac{1}{2}, \frac{1}{2}\right)$ & {$\left[\begin{array}{ll}03 & 12 \\
-i] & (+) \mid[-[-]\end{array}\right.$} & {$[-] \mid(-)(-)\left[\begin{array}{l}12 \\
{[-}\end{array}[-i]\right.$} & $-\frac{i}{2}$ & $\frac{1}{2}$ & $-\frac{1}{2}$ & -1 & $\frac{i}{2}$ & $\frac{1}{2}$ & $\frac{1}{2}$ \\
\hline$a 4$ & $\left(-\frac{1}{2},-\frac{1}{2}\right)$ & $\left(\begin{array}{l}03 \\
(+i)\end{array}[-] \mid[-[-]\right.$ & {$[-] \mid[-](-i)$} & $\frac{i}{2}$ & $-\frac{1}{2}$ & $-\frac{1}{2}$ & -1 & $\frac{i}{2}$ & $\frac{1}{2}$ & $\frac{1}{2}$ \\
\hline$b 1$ & $\left(\frac{1}{2}, \frac{1}{2}\right)$ & $\begin{array}{ccc}03 & 12 & 56 \\
{[+i]} & {[+]} & (+)\end{array}$ & 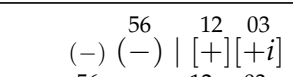 & $\frac{i}{2}$ & $\frac{1}{2}$ & $\frac{1}{2}$ & 1 & $-\frac{i}{2}$ & $-\frac{1}{2}$ & $\frac{1}{2}$ \\
\hline$b_{2}$ & $\left(\frac{1}{2},-\frac{1}{2}\right)$ & $\left(\begin{array}{ll}03 & 12 \\
(-i) & (-)\end{array} \mid(+)\right.$ & $(-) \stackrel{56}{(-)} \mid(-){ }^{12}(+)(+3)$ & $-\frac{i}{2}$ & $-\frac{1}{2}$ & $\frac{1}{2}$ & 1 & $-\frac{i}{2}$ & $-\frac{1}{2}$ & $\frac{1}{2}$ \\
\hline$b 3$ & $\left(-\frac{1}{2}, \frac{1}{2}\right)$ & 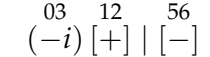 & {$\left[\begin{array}{ll}56 \\
{[-] \mid}\end{array} \mid \begin{array}{ll}12 & 03 \\
+ & 03\end{array}\right.$} & $-\frac{i}{2}$ & $\frac{1}{2}$ & $-\frac{1}{2}$ & -1 & $-\frac{i}{2}$ & $-\frac{1}{2}$ & $\frac{1}{2}$ \\
\hline$b 4$ & $\left(-\frac{1}{2},-\frac{1}{2}\right)$ & {$\left[\begin{array}{ll}03 & 12 \\
{[+i]} & (-) \mid[-]\end{array}\right.$} & {$\left[\begin{array}{ll}56 & 12 \\
{[-] \mid(-)} & (+)[+i]\end{array}\right.$} & $\frac{i}{2}$ & $-\frac{1}{2}$ & $-\frac{1}{2}$ & -1 & $-\frac{i}{2}$ & $-\frac{1}{2}$ & $\frac{1}{2}$ \\
\hline c 1 & $\left(\frac{1}{2}, \frac{1}{2}\right)$ & 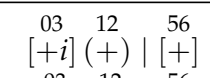 & 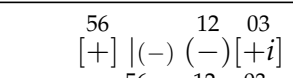 & $\frac{i}{2}$ & $\frac{1}{2}$ & $\frac{1}{2}$ & 1 & $-\frac{i}{2}$ & $\frac{1}{2}$ & $-\frac{1}{2}$ \\
\hline$c 2$ & $\left(\frac{1}{2},-\frac{1}{2}\right)$ & $\left(\begin{array}{ll}03 & 12 \\
(-i) & {[-}\end{array}\right] \mid\left[\begin{array}{l}56 \\
+\end{array}\right]$ & {$\left[\begin{array}{l}56 \\
{[+] \mid\left[\begin{array}{l}12 \\
-\end{array}\right](+3)}\end{array}\right.$} & $-\frac{i}{2}$ & $-\frac{1}{2}$ & $\frac{1}{2}$ & 1 & $-\frac{i}{2}$ & $\frac{1}{2}$ & $-\frac{1}{2}$ \\
\hline$c 3$ & $\left(-\frac{1}{2}, \frac{1}{2}\right)$ & $\left(\begin{array}{ll}03 & 12 \\
(-i) & (+)\end{array} \mid\left(\begin{array}{l}56 \\
-\end{array}\right)\right.$ & $(-) \stackrel{56}{(+)} \mid(-)\left(\begin{array}{l}12 \\
(-)\end{array}\right)(+3)$ & $-\frac{i}{2}$ & $\frac{1}{2}$ & $-\frac{1}{2}$ & -1 & $-\frac{i}{2}$ & $\frac{1}{2}$ & $-\frac{1}{2}$ \\
\hline$c 4$ & $\left(-\frac{1}{2},-\frac{1}{2}\right)$ & 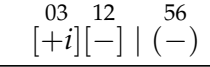 & 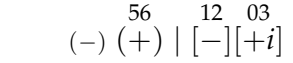 & $\frac{i}{2}$ & $-\frac{1}{2}$ & $-\frac{1}{2}$ & -1 & $-\frac{i}{2}$ & $\frac{1}{2}$ & $-\frac{1}{2}$ \\
\hline$d 1$ & $\left(\frac{1}{2}, \frac{1}{2}\right)$ & $\begin{array}{ccc}03 & 12 & 56 \\
(+i) & {[+] \mid[+]}\end{array}$ & 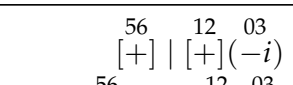 & $\frac{i}{2}$ & $\frac{1}{2}$ & $\frac{1}{2}$ & 1 & $\frac{i}{2}$ & $-\frac{1}{2}$ & $-\frac{1}{2}$ \\
\hline$d 2$ & $\left(\frac{1}{2},-\frac{1}{2}\right)$ & {$\left[\begin{array}{ll}03 & 12 \\
-i & (-)\end{array} \mid\left[+\left[\begin{array}{l}56 \\
+\end{array}\right.\right.\right.$} & $\begin{array}{l}56 \\
{[+] \mid(-)(+)}\end{array}$ & $-\frac{i}{2}$ & $-\frac{1}{2}$ & $\frac{1}{2}$ & 1 & $\frac{i}{2}$ & $-\frac{1}{2}$ & $-\frac{1}{2}$ \\
\hline$d 3$ & $\left(-\frac{1}{2}, \frac{1}{2}\right)$ & {$\left[\begin{array}{ll}03 & 12 \\
{[-i]} & {[+] \mid(-)}\end{array}\right.$} & $\left.(-) \stackrel{56}{56}^{+}\right) \mid\left[\begin{array}{l}12 \\
+\end{array}\right][-i]$ & $-\frac{i}{2}$ & $\frac{1}{2}$ & $-\frac{1}{2}$ & -1 & $\frac{i}{2}$ & $-\frac{1}{2}$ & $-\frac{1}{2}$ \\
\hline$d 4$ & $\left(-\frac{1}{2},-\frac{1}{2}\right)$ & 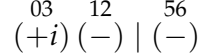 & $(-) \stackrel{56}{(+)} \mid(-)\left(\begin{array}{l}12 \\
(+)\end{array}\left(\begin{array}{l}03 \\
-i\end{array}\right)\right.$ & $\frac{i}{2}$ & $-\frac{1}{2}$ & $-\frac{1}{2}$ & -1 & $\frac{i}{2}$ & $-\frac{1}{2}$ & $-\frac{1}{2}$ \\
\hline
\end{tabular}

Half of vectors, the eigenvectors of the Cartan subalgebra, Equation (6), which are products of nilpotents and projectors, are odd products of $\gamma^{a \prime}$ s and half of them are even products of $\gamma^{a \prime}$ s. On Table 1 only Clifford odd vectors are presented.

Let us make a choice of the vacuum state $[5,6,10,11]$. (In the case of a general even $d$ the normalization factor is $\frac{1}{\sqrt{2^{\frac{d}{2}-1}}}$, since the vacuum states, generated by projectors only, follows from the starting products

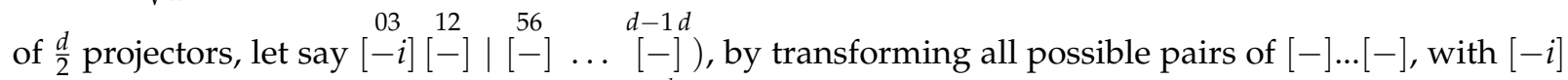
included, to $[+] \ldots[+]$, creating therefore $2^{\frac{d}{2}-1}$ summands, which is in the case of $d+(5+1)$ chosen as follows

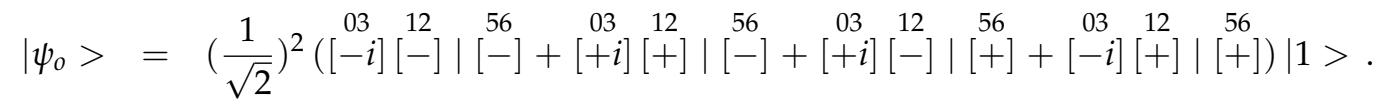


The reader can check, taking into account Equations (1) and (15) or Equation (10) (taken from Reference [3])

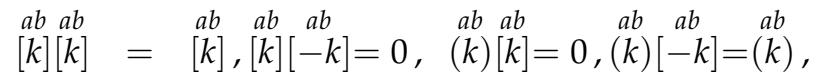

that

$$
\begin{aligned}
\hat{b}_{m}^{f \dagger} \mid \psi_{o}>: & =\mid \psi_{m}^{f}>, \\
\hat{b}_{m}^{f} \mid \psi_{o}> & =0 \cdot \mid \psi_{o}>, \\
\left\{\hat{b}_{m}^{f \dagger}, \hat{b}_{m^{\prime}}^{f^{\prime}}\right\}_{+} \mid \psi_{o}> & =\delta^{f f^{\prime}} \delta_{m m^{\prime}} \mid \psi_{o}>, \\
\left\{\hat{b}_{m}^{f \dagger}, \hat{b}_{m^{\prime}}^{f^{\prime}+}\right\}_{+} & =0 \cdot \mid \psi_{o}>, \\
\left\{\hat{b}_{m}^{f}, \hat{b}_{m^{\prime}}^{f^{\prime}}\right\}_{+} & =0 \cdot \mid \psi_{o}>, \\
\forall m \text { and } \forall f . &
\end{aligned}
$$

The relations among creation and annihilation operators in Equation (11) fulfill all the Dirac's requirements for the second quantized fermions.

\subsection{Action}

The Lorentz invariant action for a free massless fermion, describing internal degrees of freedom in Clifford space, is well known

$$
\mathcal{A}_{f}=\int d^{d} x \frac{1}{2}\left(\psi^{\dagger} \gamma^{0} \gamma^{a} p_{a} \psi\right)+\text { h.c. }
$$

$p_{a}=i \frac{\partial}{\partial x^{a}}$. It leads to the Weyl equations of motion

$$
\gamma^{a} p_{a} \mid \psi>=0
$$

which fulfill also the Klein-Gordon equation

$$
\gamma^{a} p_{a} \gamma^{b} p_{b}\left|\psi>=p^{a} p_{a}\right| \psi>=0,
$$

$\gamma^{0}$ appears in the action to take care of the Lorentz invariance of the action.

To illustrate that fermions interacting with only gravity in $d=(13+1)$-dimensional space manifest charges in $d=(3+1)$, we present in Appendix A the whole action used in the spin-charge-family, in which fermions interact with the vielbeins and the two kinds of the spin connections fields, the gauge fields of momenta $p^{a}$ and the two kinds of the generators of the Lorentz transformations in the internal space of fermions $S^{a b}$ and $\tilde{S}^{a b}$, respectively. We comment there the part of the action, which manifest in $d=(3+1)$ all the observed vector gauge fields, the scalar gauge fields, offering explanation for the scalar higgs and Yukawa couplings of the standard model, explaining as well several phenomena in physics of elementary fermion and boson fields and in cosmology. Some of the achievements of this theory is also presented.

Solutions of equations of motion, Equation (13), for a free massless fermion with momentum $p^{a}=$ $\left(\left|p^{0}\right|, p^{1}, p^{2}, p^{3}, 0,0\right)$ and a particular charge $\frac{1}{2}$, are for any family $f$ superposition of basis vectors $\left|\psi_{f}^{m}\right\rangle=$ $\hat{b}_{m}^{f \dagger} \mid \psi_{0}>$ with spin $\frac{1}{2}$ and spin $-\frac{1}{2}$, both multiplied by $e^{-i\left(p^{0} x^{0}-\vec{p} \vec{x}\right)}$, (see Equation (97) in Reference [10]). Coefficients in the superposition depend on the momentum $p^{a}$. 


\subsection{Creation and Annihilation Operators in $d=(3+1)$ Space Embedded in $d=(5+1)$ Space}

The creation and annihilation operators of Table 1 are all of an odd Clifford character (they are superposition of odd products of $\gamma^{a \prime}$ ). The rest of the $2^{4}$ creation operators of an even Clifford character can be found in References [10,11].

Taking into account Equation (1) one recognizes that $\gamma^{a \text { 's }}$ transform $\stackrel{a b}{(k)}$ into $\stackrel{a b}{[-k]}$, never to $[k]$, while $\tilde{\gamma}^{a \prime}$ s transform $\stackrel{a b}{(k)}$ into $[k]$, never to $[-k]$

$$
\begin{aligned}
& \gamma^{a} \stackrel{a b}{(k)}=\eta^{a a}[-k], \gamma^{b} \stackrel{a b}{(k)}=-i k[-k], \gamma^{a b} \stackrel{a b}{[k]}=(-k), \gamma^{b}\left[\begin{array}{c}
a b \\
{[k]}
\end{array}=-i k \eta^{a a}(\stackrel{a b}{(-k)},\right. \\
& \tilde{\gamma}^{a} \stackrel{a b}{(k)}=-i \eta^{a a}\left[\stackrel{a b}{[k]}, \tilde{\gamma^{b}} \stackrel{a b}{(k)}=-k \stackrel{a b}{[k]}, \tilde{\gamma^{a}}\left[\stackrel{a b}{[k]}=i \stackrel{a b}{(k)}, \tilde{\gamma}^{b} \stackrel{a b}{[k]}=-k \eta^{a a} \stackrel{a b}{(k)}\right. \text {. }\right.
\end{aligned}
$$

With the knowledge presented in Equation (15) it is not difficult to reproduce Table 2, representing vectors that belong to $d=(3+1)$ space. Vectors carry no charge and have either an odd or an even Clifford character. Multiplying these vectors by the appropriate nilpotent or projector representing the charge (that is by either the nilpotent which is the eigenstate of $S^{56}$, if the $d=(3+1)$ part has an even Clifford character, or the corresponding projector, if the $d=(3+1)$ part has an odd Clifford character) we end up with the Clifford odd vectors from Table 1 , representing four $\left(2^{\frac{6}{2}-1}\right)$ families with four $\left(2^{\frac{6}{2}-1}\right)$ members each.

The properties of vectors of Table 2 are analyzed in details in order that the correspondence with the Dirac's $\gamma$ matrices in $d=(3+1)$ space is easy to be recognized. Superposition of vectors with the spin $\pm \frac{1}{2}$ (either Clifford even or odd) solve the equations of motion, Equation (13), for free massless fermions.

As seen in Table $2 \gamma^{a \prime}$ s change the handedness (Equation (8)) $\Gamma^{(3+1)}$ of vectors, while $\tilde{\gamma}^{a \prime}$ s change the handedness $\tilde{\Gamma}^{(3+1)}$ of vectors. Both change the Clifford character of states, from Clifford odd character to Clifford even character or vice versa. $S^{a b}$, which do not belong to Cartan subalgebra, generate all the states of one representation of particular handedness $\Gamma^{(3+1)}$ and particular family quantum number. $\tilde{S}^{a b}$, which do not belong to Cartan subalgebra, transform a family member of one family into the same family member number of another family, $\tilde{\gamma}^{a}$ change the family quantum number as well as the handedness $\tilde{\Gamma}^{(3+1)}$.

Dirac matrices $\gamma^{a}$ and $S^{a b}$ do not distinguish among the families: Corresponding family members of any family have the same properties with respect to $S^{a b}$ and $\gamma^{a}$, manifesting for $d=(3+1)$ space four times twice $2 \times 2$ by diagonal matrices, which are, up to a phase, identical. The operators $\gamma^{a}$ and $S^{a b}$ are correspondingly four times $4 \times 4$ matrices.

One finds among Clifford even vectors of Table 2 the ones which are products of projectors; they are Hermitian self conjugated. The remaining even vectors are Hermitian conjugated to each other $\left(\hat{b}_{1}^{a+}\right.$ is Hermitian conjugated to $\hat{b}_{2}^{d \dagger}$, for example). In the Clifford odd part of Table 2 one finds that $\hat{b}_{m=(3,4)}^{a \dagger}$

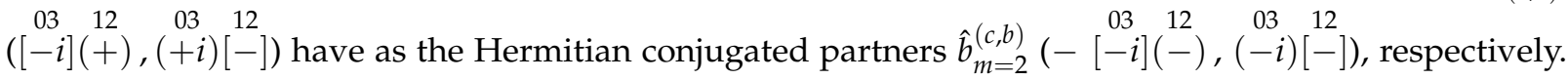

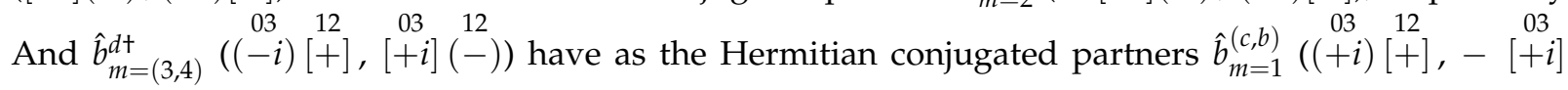
12

$(+))$, respectively.

The vacuum state for the $d=(3+1)$ case is correspondingly:

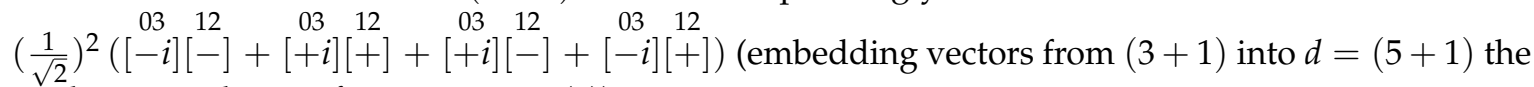
vacuum becomes the one from Equation (9)). 
Embedding $\hat{b}_{m+}^{b+} \quad \begin{array}{lll}03 & 12\end{array}$ manifesting in $d=(3+1)$ the charge $-\frac{1}{2}$ (while the annihilation operator extends into $\left.-[-i](-)[-]\right)$.

Table 2. In this table $2^{d}=16$ vectors, describing internal space of fermions in $d=(3+1)$, are presented. Each vector carries the family member quantum number $m=(1,2,3,4)$-determined by $S^{03}$ and $S^{12}$, Equations (6) and (7)-and the family quantum number $f=(a, b, c, d)$-determined by $\tilde{S}^{03}$ and $\tilde{S}^{12}$, Equations (6) and (7). Vectors $\psi_{m}^{f}$ are obtained by applying $\hat{b}_{m}^{f \dagger}$ on the vacuum state, Equation (9). Vectors, that is the family members of any family, split into even (they are sums of products of an even number of $\gamma^{a}$ s) and odd (they are sums of products of an odd number of $\gamma^{a \prime}$ ). If these vectors are embedded into the vectors of $d=(5+1)$ (by being multiplied by an appropriate nilpotent or projector so that they are of an odd Clifford character), they "gain" charges as presented in Table 1.

\begin{tabular}{|c|c|c|c|c|c|c|c|c|c|c|c|c|c|c|c|}
\hline & $\psi_{m}^{f}$ & $\gamma_{0} \psi_{m}^{f}$ & $\gamma_{1} \psi_{m}^{f}$ & $\gamma_{2} \psi_{m}^{f}$ & $\gamma_{3} \psi_{m}^{f}$ & $\tilde{\gamma}_{0} \psi_{m}^{f}$ & $\tilde{\gamma}_{1} \psi_{m}^{f}$ & $\tilde{\gamma}_{2} \psi_{m}^{f}$ & $\tilde{\gamma}_{3} \psi_{m}^{f}$ & $S^{03}$ & $S^{12}$ & $\tilde{S}^{03}$ & $\tilde{S}^{12}$ & $\Gamma^{(3+1)}$ & $\tilde{\Gamma}^{(3+1)}$ \\
\hline $\begin{array}{l}\psi_{1}^{a} \\
\psi_{2}^{a} \\
\psi_{3}^{a} \\
\psi_{4}^{a} \\
\end{array}$ & $\begin{array}{l}(+i)(+) \\
{[-i][-]} \\
{[-i](+)} \\
(+i)[-] \\
\end{array}$ & $\begin{array}{l}\psi_{3}^{a} \\
\psi_{4}^{a} \\
\psi_{1}^{a} \\
\psi_{2}^{a} \\
\end{array}$ & $\begin{array}{r}\psi_{4}^{a} \\
\psi_{3}^{a} \\
-\psi_{2}^{a} \\
-\psi_{1}^{a} \\
\end{array}$ & $\begin{array}{r}i \psi_{4}^{a} \\
-i \psi_{3}^{a} \\
-i \psi_{2}^{a} \\
i \psi_{1}^{a} \\
\end{array}$ & $\begin{array}{r}\psi_{3}^{a} \\
-\psi_{4}^{a} \\
-\psi_{1}^{a} \\
\psi_{2}^{a} \\
\end{array}$ & $\begin{array}{r}-i \psi_{1}^{b} \\
i \psi_{2}^{b} \\
i \psi_{3}^{b} \\
-i \psi_{4}^{b} \\
\end{array}$ & $\begin{array}{r}-i \psi_{1}^{c} \\
i \psi_{2}^{c} \\
i \psi_{3}^{c} \\
-i \psi_{4}^{c} \\
\end{array}$ & $\begin{array}{r}\psi_{1}^{c} \\
-\psi_{2}^{c} \\
-\psi_{3}^{c} \\
\psi_{4}^{c} \\
\end{array}$ & $\begin{array}{r}-i \psi_{1}^{b} \\
i \psi_{2}^{b} \\
i \psi_{3}^{b} \\
-i \psi_{4}^{b} \\
\end{array}$ & $\begin{array}{r}\frac{i}{2} \\
-\frac{i}{2} \\
-\frac{i}{2} \\
\frac{i}{2} \\
\end{array}$ & $-\overline{2}$ & $\begin{array}{l}\frac{i}{2} \\
\frac{i}{2} \\
\frac{i}{2} \\
\frac{i}{2} \\
\end{array}$ & $\frac{1}{2}$ & $\begin{array}{r}1 \\
1 \\
-1 \\
-1\end{array}$ & $\begin{array}{l}1 \\
1 \\
1 \\
1\end{array}$ \\
\hline $\begin{array}{l}\psi_{1}^{b} \\
\psi_{2}^{b} \\
\psi_{3}^{b} \\
\psi_{4}^{b} \\
\end{array}$ & $\begin{array}{l}{[+i](+)} \\
(-i)[-] \\
(-i)(+) \\
{[+i][-]}\end{array}$ & $\begin{array}{l}\psi_{3}^{b} \\
\psi_{4}^{b} \\
\psi_{1}^{b} \\
\psi_{2}^{b} \\
\end{array}$ & $\begin{array}{r}-\psi_{4}^{b} \\
-\psi_{3}^{b} \\
\psi_{2}^{b} \\
\psi_{1}^{b} \\
\end{array}$ & $\begin{array}{r}-i \psi_{4}^{b} \\
i \psi_{3}^{b} \\
i \psi_{2}^{b} \\
-i \psi_{1}^{b} \\
\end{array}$ & $\begin{array}{r}\psi_{3}^{b} \\
-\psi_{4}^{b} \\
-\psi_{1}^{b} \\
\psi_{2}^{b} \\
\end{array}$ & $\begin{array}{r}i \psi_{1}^{a} \\
-i \psi_{2}^{a} \\
-i \psi_{3}^{a} \\
i \psi_{4}^{a} \\
\end{array}$ & $\begin{array}{r}i \psi_{1}^{d} \\
-i \psi_{2}^{d} \\
-i \psi_{3}^{d} \\
i \psi_{4}^{d} \\
\end{array}$ & $\begin{array}{r}-\psi_{1}^{d} \\
\psi_{2}^{d} \\
\psi_{3}^{d} \\
-\psi_{4}^{d} \\
\end{array}$ & $\begin{array}{r}-i \psi_{1}^{a} \\
i \psi_{2}^{a} \\
i \psi_{3}^{a} \\
-i \psi_{4}^{a} \\
\end{array}$ & $\begin{array}{r}\frac{i}{2} \\
-\frac{i}{2} \\
-\frac{i}{2} \\
\frac{i}{2} \\
\end{array}$ & $\begin{array}{r}\frac{1}{2} \\
-\frac{1}{2} \\
\frac{1}{2} \\
-\frac{1}{2} \\
\end{array}$ & $\begin{array}{l}-\frac{i}{2} \\
-\frac{i}{2} \\
-\frac{i}{2} \\
-\frac{i}{2} \\
\end{array}$ & $\begin{array}{l}\frac{1}{2} \\
\frac{1}{2} \\
\frac{1}{1}\end{array}$ & $\begin{array}{r}1 \\
1 \\
-1 \\
-1 \\
\end{array}$ & $\begin{array}{l}-1 \\
-1 \\
-1 \\
-1\end{array}$ \\
\hline $\begin{array}{l}\psi_{1}^{c} \\
\psi_{2}^{c} \\
\psi_{3}^{c} \\
\psi_{4}^{c}\end{array}$ & $\begin{array}{l}(+i)[+] \\
{[-i](-)} \\
{[-i][+]} \\
(+i)(-)\end{array}$ & $\begin{array}{l}\psi_{3}^{c} \\
\psi_{4}^{c} \\
\psi_{1}^{c} \\
\psi_{2}^{c}\end{array}$ & $\begin{array}{r}-\psi_{4}^{c} \\
-\psi_{3}^{c} \\
\psi_{2}^{c} \\
\psi_{1}^{c}\end{array}$ & $\begin{array}{r}-i \psi_{4}^{c} \\
i \psi_{3}^{c} \\
i \psi_{2}^{c} \\
-i \psi_{1}^{c}\end{array}$ & $\begin{array}{r}\psi_{3}^{c} \\
-\psi_{4}^{c} \\
-\psi_{1}^{c} \\
\psi_{2}^{c}\end{array}$ & $\begin{array}{r}i \psi_{1}^{d} \\
-i \psi_{2}^{d} \\
-i \psi_{3}^{d} \\
i \psi_{4}^{d}\end{array}$ & $\begin{array}{r}-i \psi_{1}^{a} \\
i \psi_{2}^{a} \\
i \psi_{3}^{a} \\
-i \psi_{4}^{a}\end{array}$ & $\begin{array}{r}-\psi_{1}^{a} \\
\psi_{2}^{a} \\
\psi_{3}^{a} \\
-\psi_{4}^{a}\end{array}$ & $\begin{array}{r}i \psi_{1}^{d} \\
-i \psi_{2}^{d} \\
-i \psi_{3}^{d} \\
i \psi_{4}^{d}\end{array}$ & $\begin{array}{r}\frac{i}{2} \\
-\frac{i}{2} \\
-\frac{i}{2} \\
\frac{i}{2}\end{array}$ & $\begin{array}{r}2 \\
-\frac{1}{2}\end{array}$ & $\begin{array}{l}\frac{i}{2} \\
\frac{i}{2} \\
\frac{i}{2} \\
\frac{i}{2}\end{array}$ & $\begin{array}{l}-\frac{1}{2} \\
-\frac{1}{2} \\
-\frac{1}{2} \\
-\frac{1}{2}\end{array}$ & $\begin{array}{r}1 \\
1 \\
-1 \\
-1\end{array}$ & $\begin{array}{l}-1 \\
-1 \\
-1 \\
-1\end{array}$ \\
\hline $\begin{array}{l}\psi_{1}^{d} \\
\psi_{2}^{d} \\
\psi_{3}^{d} \\
\psi_{4}^{d}\end{array}$ & $\begin{array}{l}{[+i][+]} \\
(-i)(-) \\
(-i)[+] \\
{[+i](-)}\end{array}$ & $\begin{array}{l}\psi_{3}^{d} \\
\psi_{4}^{d} \\
\psi_{1}^{d} \\
\psi_{2}^{d}\end{array}$ & $\begin{array}{r}\psi_{4}^{d} \\
\psi_{3}^{d} \\
-\psi_{2}^{d} \\
-\psi_{1}^{d}\end{array}$ & $\begin{array}{r}i \psi_{4}^{d} \\
-i \psi_{3}^{d} \\
-i \psi_{2}^{d} \\
i \psi_{1}^{d}\end{array}$ & $\begin{array}{r}\psi_{3}^{d} \\
-\psi_{4}^{d} \\
-\psi_{1}^{d} \\
\psi_{2}^{d}\end{array}$ & $\begin{array}{r}-i \psi_{1}^{c} \\
i \psi_{2}^{c} \\
i \psi_{3}^{c} \\
-i \psi_{4}^{c}\end{array}$ & $\begin{array}{r}i \psi_{1}^{b} \\
-i \psi_{2}^{b} \\
-i \psi_{3}^{b} \\
i \psi_{4}^{b}\end{array}$ & $\begin{array}{r}\psi_{1}^{b} \\
-\psi_{2}^{b} \\
-\psi_{3}^{b} \\
\psi_{4}^{b}\end{array}$ & $\begin{array}{r}i \psi_{1}^{c} \\
-i \psi_{2}^{c} \\
-i \psi_{3}^{c} \\
i \psi_{4}^{c}\end{array}$ & $\begin{array}{r}\frac{i}{2} \\
-\frac{i}{2} \\
-\frac{i}{2} \\
\frac{i}{2}\end{array}$ & $\begin{array}{r}\frac{1}{2} \\
-\frac{1}{2}\end{array}$ & $\begin{array}{l}-\frac{i}{2} \\
-\frac{i}{2} \\
-\frac{i}{2} \\
-\frac{i}{2}\end{array}$ & $\begin{array}{l}-\frac{1}{2} \\
-\frac{1}{2} \\
-\frac{1}{2} \\
-\frac{1}{2}\end{array}$ & $\begin{array}{r}1 \\
1 \\
-1 \\
-1\end{array}$ & $\begin{array}{l}1 \\
1 \\
1 \\
1\end{array}$ \\
\hline
\end{tabular}

\section{3. $\gamma^{a}, S^{a b}, \tilde{\gamma}^{a}$ and $\tilde{S}^{a b}$ Matrices in $d=(3+1)$}

There are $2^{d}=16$ basis vectors in $d=(3+1)$, presented in Table 2 . They all can be found as well as a part of basis vectors in Table 1, with either nilpotent or projector expressing the charge added, so that each basis vector has an odd Clifford character belonging to one of $16\left(2^{\frac{6}{2}-1} \times 2^{\frac{6}{2}-1}\right)$ odd vectors in Table 1 . Basis vectors are products of nilpotents and projectors, which are eigenstates of the Cartan subalgebra operators, Equation (6), as presented in Equations (7).

The family members of a family are reachable from any member by either $S^{a b}$ or by $\gamma^{a}$, and represent twice two vectors of definite handedness $\Gamma^{(d)}$ in $d=(3+1)$. Different families are reachable by either $\tilde{S}^{a b}$ or by $\tilde{\gamma}^{a}$. Each state carries correspondingly quantum numbers of the two kinds of the Cartan subalgebra. In Table 2 also $\Gamma^{(3+1)}\left(=-4 i S^{03} S^{12}\right)$ and $\tilde{\Gamma}^{(3+1)}\left(=-4 i \tilde{S}^{03} \tilde{S}^{12}\right)$ are presented.

Let us again point out that if we treat all the basis vectors in $d=(3+1)$ as a part of vectors in $d=(5+1)$, all of an odd Clifford character, so that they carry also a charge which is the spin $S^{56}$, then the family members of a family are reachable by $S^{a b}$ only and families by $\tilde{S}^{a b}$ only.

When the basis vectors are chosen and Table 2 is made, it is not difficult to find the matrix representations for the operators $\left(\gamma^{a}, S^{a b}, \tilde{\gamma}^{a}, \tilde{S}^{a b}, \Gamma^{(3+1)}, \tilde{\Gamma}^{(3+1)}\right)$. They are obviously $16 \times 16$ matrices with a $4 \times 4$ diagonal or off diagonal or partly diagonal and partly off diagonal substructure.

Dirac matrices pay attention on only one family, any one, and do not take into account charges. 
Let us define, to simplify the notation, the unit $4 \times 4$ submatrix and the submatrix with all the matrix elements equal to zero as follows

$$
\mathbf{1}=\left(\begin{array}{ll}
1 & 0 \\
0 & 1
\end{array}\right)=\sigma^{0}, \quad \mathbf{0}=\left(\begin{array}{ll}
0 & 0 \\
0 & 0
\end{array}\right) .
$$

We also use $(2 \times 2)$ Pauli matrices

$$
\sigma^{1}=\left(\begin{array}{ll}
0 & 1 \\
1 & 0
\end{array}\right), \quad \sigma^{2}=\left(\begin{array}{cc}
0 & -i \\
i & 0
\end{array}\right), \quad \sigma^{3}=\left(\begin{array}{cc}
1 & 0 \\
0 & -1
\end{array}\right) .
$$

It is easy to find the matrix representations for $\gamma^{0}, \gamma^{1}, \gamma^{2}$ and $\gamma^{3}$ from Table 2

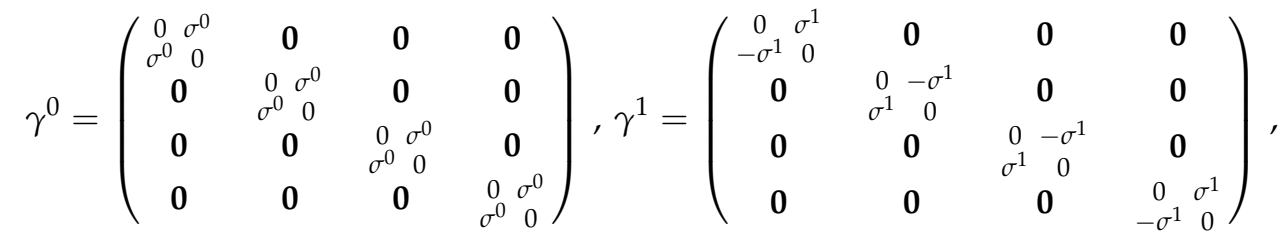

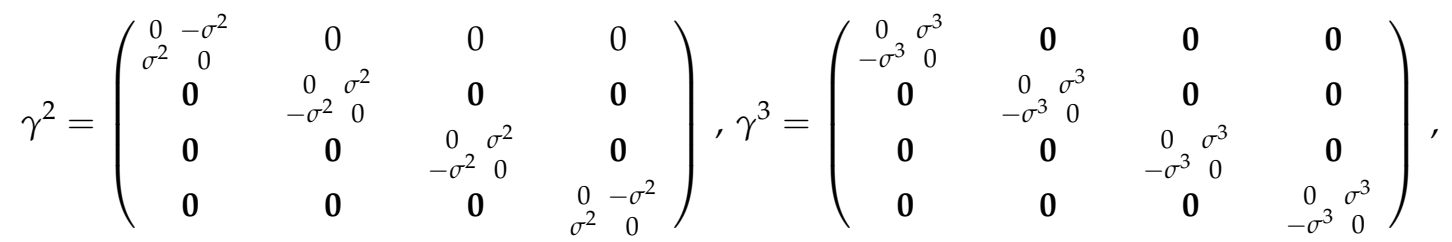

manifesting the $4 \times 4$ substructure (of Dirac matrices) along the diagonal of $16 \times 16$ matrices.

The representations of $\tilde{\gamma}^{a}$ do not appear in the Dirac case. They manifest the off diagonal structure as follows

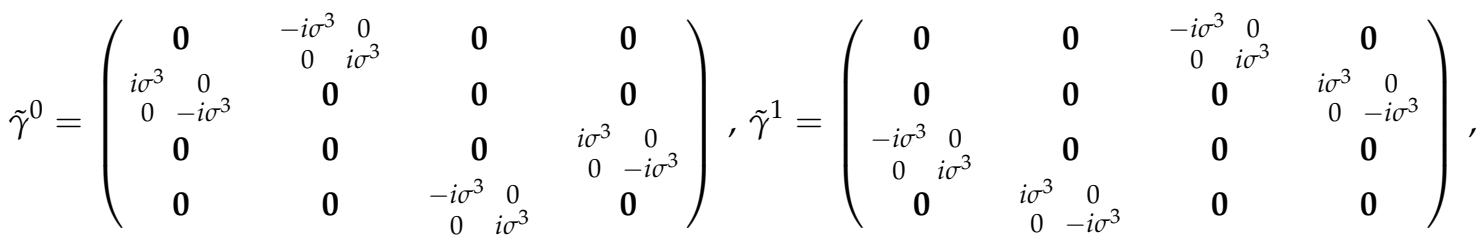

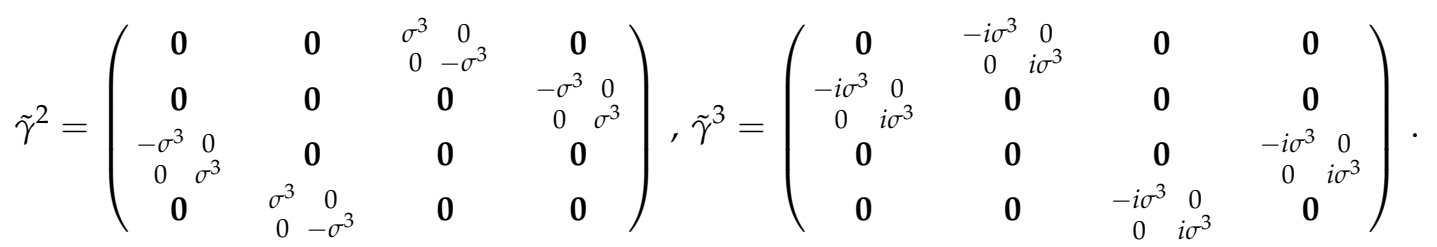

Matrices $S^{a b}$ have again along the diagonal the $4 \times 4$ substructure, repeating, up to a phase, as expected the corresponding Dirac matrices, since the Dirac $S^{a b}$ do not distinguish among families.

$$
S^{01}=\frac{i}{2}\left(\begin{array}{cccccc}
\sigma^{1} & 0 & \mathbf{0} & \mathbf{0} & \mathbf{0} \\
0 & -\sigma^{1} & -\sigma^{1} & 0 & \mathbf{0} & \mathbf{0} \\
\mathbf{0} & 0 & \sigma^{1} & -\sigma^{1} & 0 & \mathbf{0} \\
\mathbf{0} & \mathbf{0} & 0 & \sigma^{1} & \mathbf{0} \\
\mathbf{0} & \mathbf{0} & \mathbf{0} & \sigma^{1} & 0 \\
0 & 0 & -\sigma^{1}
\end{array}\right), S^{02}=\frac{i}{2}\left(\begin{array}{cccccc}
-\sigma^{2} & 0 & \mathbf{0} & \mathbf{0} & \mathbf{0} \\
0 & \sigma^{2} & \sigma^{2} & 0 & \mathbf{0} & \mathbf{0} \\
\mathbf{0} & 0 & -\sigma^{2} & \sigma^{2} & 0 & \mathbf{0} \\
\mathbf{0} & \mathbf{0} & 0-\sigma^{2} & -\sigma^{2} & 0 \\
\mathbf{0} & \mathbf{0} & \mathbf{0} & 0 & \sigma^{2}
\end{array}\right),
$$




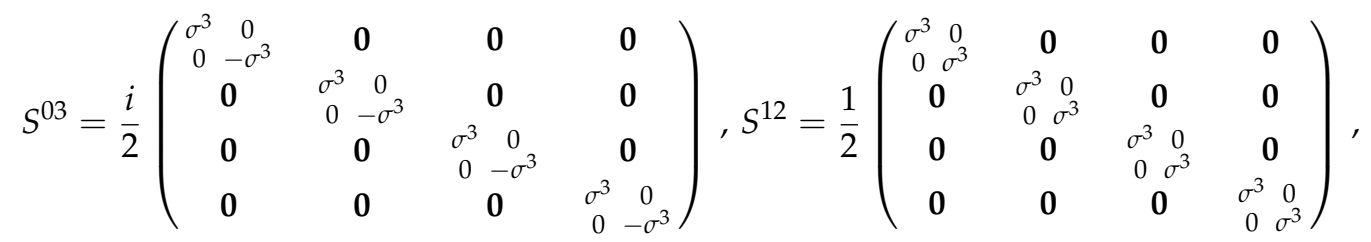

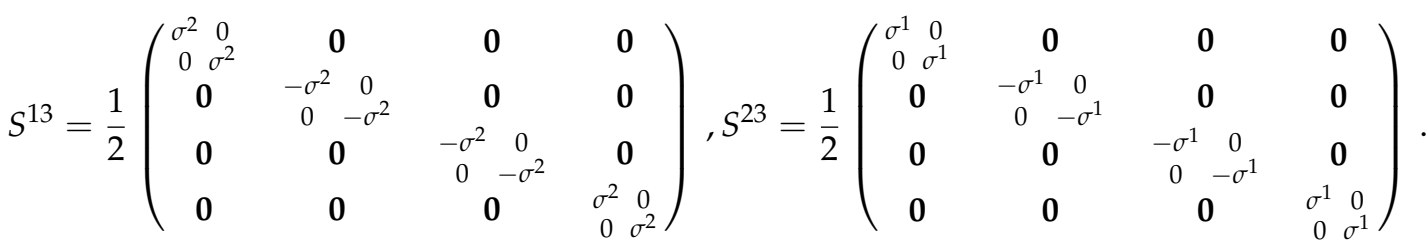

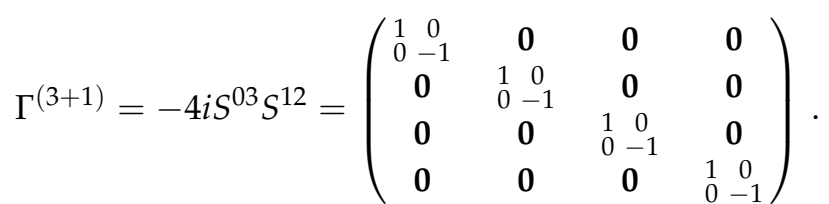

The operators $\tilde{S}^{a b}$ have again off diagonal $4 \times 4$ substructure, except $\tilde{S}^{03}$ and $\tilde{S}^{12}$, which are diagonal.

$$
\begin{gathered}
\tilde{S}^{01}=-\frac{i}{2}\left(\begin{array}{cccc}
\mathbf{0} & \mathbf{0} & \mathbf{0} & \mathbf{1} \\
\mathbf{0} & \mathbf{0} & \mathbf{1} & \mathbf{0} \\
\mathbf{0} & \mathbf{1} & \mathbf{0} & \mathbf{0} \\
\mathbf{1} & \mathbf{0} & \mathbf{0} & \mathbf{0}
\end{array}\right), \tilde{S}^{02}=\frac{1}{2}\left(\begin{array}{cccc}
\mathbf{0} & \mathbf{0} & \mathbf{0} & \mathbf{1} \\
\mathbf{0} & \mathbf{0} & \mathbf{1} & \mathbf{0} \\
\mathbf{0} & -\mathbf{1} & \mathbf{0} & \mathbf{0} \\
-\mathbf{1} & \mathbf{0} & \mathbf{0} & \mathbf{0}
\end{array}\right), \tilde{S}^{03}=\frac{i}{2}\left(\begin{array}{cccc}
\mathbf{1} & \mathbf{0} & \mathbf{0} & \mathbf{0} \\
\mathbf{0} & -\mathbf{1} & \mathbf{0} & \mathbf{0} \\
\mathbf{0} & \mathbf{0} & \mathbf{1} & \mathbf{0} \\
\mathbf{0} & \mathbf{0} & \mathbf{0} & -\mathbf{1}
\end{array}\right) \\
\tilde{S}^{12}=\frac{1}{2}\left(\begin{array}{ccccc}
\mathbf{1} & \mathbf{0} & \mathbf{0} & \mathbf{0} \\
\mathbf{0} & \mathbf{1} & \mathbf{0} & \mathbf{0} \\
\mathbf{0} & \mathbf{0} & -\mathbf{1} & \mathbf{0} \\
\mathbf{0} & \mathbf{0} & \mathbf{0} & -\mathbf{1}
\end{array}\right), \tilde{S}^{13}=\frac{i}{2}\left(\begin{array}{cccc}
\mathbf{0} & \mathbf{0} & \mathbf{0} & -\mathbf{1} \\
\mathbf{0} & \mathbf{0} & \mathbf{1} & \mathbf{0} \\
\mathbf{0} & -\mathbf{1} & \mathbf{0} & \mathbf{0} \\
\mathbf{1} & \mathbf{0} & \mathbf{0} & \mathbf{0}
\end{array}\right), \tilde{S}^{23}=\frac{1}{2}\left(\begin{array}{cccc}
\mathbf{0} & \mathbf{0} & \mathbf{0} & -\mathbf{1} \\
\mathbf{0} & \mathbf{0} & \mathbf{1} & \mathbf{0} \\
\mathbf{0} & \mathbf{1} & \mathbf{0} & \mathbf{0} \\
-\mathbf{1} & \mathbf{0} & \mathbf{0} & \mathbf{0}
\end{array}\right) \\
\tilde{\Gamma}^{(3+1)}=-4 i \tilde{S}^{03} \tilde{S}^{12}=\left(\begin{array}{cccc}
\mathbf{1} & \mathbf{0} & \mathbf{0} & \mathbf{0} \\
\mathbf{0} & -\mathbf{1} & \mathbf{0} & \mathbf{0} \\
\mathbf{0} & \mathbf{0} & -\mathbf{1} & \mathbf{0} \\
\mathbf{0} & \mathbf{0} & \mathbf{0} & \mathbf{1}
\end{array}\right) .
\end{gathered}
$$

\section{Conclusions}

In this contribution the internal degrees of freedom of fermions are represented by the Clifford algebra objects, which offers not only the description of spins and charges as it is in the case of the Dirac $2 \cdot 2^{\frac{d}{2}-1}$ matrices in $d=(3+1)$ space but also the description of $2 \cdot 2^{\frac{d}{2}-1}$ families.

Embedding $(3+1)$ space into $(2 n+1)$-dimensional space the Clifford algebra offers as well the description of charges.

This contribution manifests:

i. That the appearance of two kinds of the Clifford algebra objects, $\gamma^{a \prime}$ s and $\tilde{\gamma}^{a \prime}$ s, offers the explanation for the appearance of families of quarks and leptons, without postulating the family groups.

ii. The appearance of charges in $d=(3+1)$ part if $d \geq(5+1)$. To reproduce all the properties of fermions manifesting as quarks and leptons in $d=(3+1)$ the dimension of space time must be $d \geq(13+1)$. 
This contribution is a part of the project of (N.S.M.B.), named the spin-charge-family theory ([1-11] and the references therein), which uses the Clifford algebra to describe the internal degrees of freedom of fermions, proving that the theory offers the next step to the standard model.

The theory suggests that since the Clifford algebra in $d \geq(13+1)$ offers in $d=(3+1)$ the description of spins, handedness, and all the charges of quarks and leptons, the gravity and all the vector and scalar gauge fields must be of the same origin, determined by the vielbeins and the two kinds of the spin connection fields, the gauge fields of momenta, of $S^{a b}$ and of $\tilde{S}^{a b}$.

We present in this contribution the matrix representations of operators, $\gamma^{a \prime}$ s, $S^{a b}$ 's, $\tilde{\gamma}^{a}$ 's, $\tilde{S}^{a b}$ 's, applying on the basis vectors, which are odd products of the Clifford objects defining the creation and annihilation operators for fermions in $d$-dimensional Clifford space, where $d=2(2 n+1)$, or $4 n, n=1$. We make a choice of $d=(3+1)$ and $d=(5+1)$, paying attention that creation and annihilation operators of fermions in $d=(3+1)$ manifest charges, if embedded in $d=(5+1)$.

Creation and annihilation operators, defining the internal space of the second quantized fermions, have an odd Clifford character (they are superposition of odd products of Clifford objects $\gamma^{a \prime}$ s). They are in our presentation products of nilpotents and projectors, chosen to be eigenvectors of the Cartan subalgebra, Equation (6), of the Lorentz algebra of $S^{a b}$, as well as of the corresponding Cartan subalgebra, Equation (6), of $\tilde{S}^{a b} . S^{a b}$ define the members of each irreducible representation of the Lorentz group, $\tilde{S}^{a b}$ define family quantum number of each irreducible representation.

Creation and annihilation operators are Hermitian conjugated to each other. We make a choice of the creation operators with respect to the annihilation operators by choosing the vacuum state, Equation (9), to be the sum of products of the annihilation operators with their Hermitian conjugated partners creation operators.

$S^{a b}$ generate $2^{\frac{d}{2}-1}$ family members of a particular family of an odd Clifford character, $\tilde{S}^{a b}$ generate the corresponding $2^{\frac{d}{2}-1}$ families. The Hermitian conjugation determines their $2^{\frac{d}{2}-1} \times 2^{\frac{d}{2}-1}$ partners (which are reachable also by $\gamma^{a} \tilde{\gamma}^{a}$ ). The Clifford even representations follow from the odd $2^{d-1}$ vectors by the application of $\gamma^{a \prime}$ s or $\tilde{\gamma}^{a \prime}$ s. There are correspondingly $2^{d}$ vectors in $d$-dimensional space $(d=2(2 n+1), 4 n)$.

The Clifford even operators, $S^{a b}$ and $\tilde{S}^{a b}$, keep the Clifford character unchanged. $\gamma^{a \prime}$ s and $\tilde{\gamma}^{a}$ 's change the Clifford character of vectors-from odd to even or vice versa.

Embedding $S O(3+1)$ into $S O(d), d>(3+1), d$ even, makes spins in $d \geq(5+1)$ to manifest in $d=(3+1)$ as charges.

One can check that the creation operators of an odd Clifford character and their Hermitian conjugated partners, applied on the vacuum state, Equation (9), fulfill the anticommutation relations for the second quantized fermions, Equation (11), postulated by Dirac, what explains the Dirac's second quantization postulates [10,11].

We demonstrate in this contribution how does the Clifford algebra reproduce the Dirac matrices in the case of one family in $d=(3+1)$ with four basis vectors and in the case of four families offered by the Clifford algebra. There are namely $2^{4}=16$ basis vectors in $d=(3+1)$ and correspondingly all the matrices have dimension $16 \times 16$, which are for the operators, determined by $\gamma^{a}$ 's, by diagonal and for the operators, determined by $\tilde{\gamma}^{a}$ 's, off diagonal, except $\tilde{S}^{03}, \tilde{S}^{12}$, which are the members of the Cartan subalgebra and correspondingly also $\tilde{\Gamma}^{(3+1)}=-4 i \tilde{S}^{03} \tilde{S}^{12}$. We keep in $d=(3+1)$ the Clifford odd and the Clifford even vectors as the basis vectors to point out, that if space of $d=(3+1)$ is embedded in $d \geq(5+1)$, all the parts, even and odd, contribute to the enlarged vector space manifesting charges.

In Appendix A we shortly illustrate some achievements and predictions of the spin-charge-family theory, using the odd Clifford algebra objects to represent internal degrees of freedom of fermions.

Author Contributions: These authors contributed equally to this work. All authors have read and agreed to the published version of the manuscript. 
Funding: This research received no external funding.

Acknowledgments: The author N.S.M.B. thanks Department of Physics, FMF, University of Ljubljana, Society of Mathematicians, Physicists and Astronomers of Slovenia, for supporting the research on the spin-charge-family theory, and Matjaž Breskvar of Beyond Semiconductor for donations, in particular for sponsoring the annual workshops entitled "What comes beyond the standard models" at Bled.

Conflicts of Interest: The authors declare no conflict of interest.

\section{Appendix A. Short Overview of Achievements of Spin-Charge-Family Theory}

One of the authors of this paper (N.S.M.B.) is proposing the spin-charge-family theory [[2-4,7-9] and references therein], in which massless fermions in $d=(13+1)$-dimensional space interact with gravity only-the vielbeins $f^{\alpha}{ }_{a}$ (the gauge fields of moments $p_{a}$ ) and the two kinds of the spin connections $\left(\omega_{a b \alpha}\right.$ and $\tilde{\omega}_{a b \alpha}$, the gauge fields of the two kinds of the Clifford algebra objects $S^{a b}$ and $\tilde{S}^{a b}$, respectively, Equations (1) and (6)).

$$
\begin{aligned}
\mathcal{A}= & \int d^{d} x E \frac{1}{2}\left(\bar{\psi} \gamma^{a} p_{0 a} \psi\right)+\text { h.c. }+ \\
& \int d^{d} x E(\alpha R+\tilde{\alpha} \tilde{R})
\end{aligned}
$$

with $p_{0 a}=f^{\alpha}{ }_{a} p_{0 \alpha}+\frac{1}{2 E}\left\{p_{\alpha}, E f^{\alpha}{ }_{a}\right\}_{-}, p_{0 \alpha}=p_{\alpha}-\frac{1}{2} S^{a b} \omega_{a b \alpha}-\frac{1}{2} \tilde{S}^{a b} \tilde{\omega}_{a b \alpha}$ and $R=\frac{1}{2}\left\{f^{\alpha[a} f^{\beta b]}\left(\omega_{a b \alpha, \beta}-\right.\right.$ $\left.\left.\omega_{c a \alpha} \omega_{b \beta}^{c}\right)\right\}+$ h.c., $\tilde{R}=\frac{1}{2}\left\{f^{\alpha[a} f^{\beta b]}\left(\tilde{\omega}_{a b \alpha, \beta}-\tilde{\omega}_{c a \alpha} \tilde{\omega}_{b \beta}^{c}\right)\right\}+$ h.c.. Here $f^{\alpha}{ }_{a}$ are inverse vielbeins to $e^{a}{ }_{\alpha}$ with the properties $e^{a}{ }_{\alpha} f_{b}^{\alpha}=\delta^{a}{ }_{b}, e^{a}{ }_{\alpha} f^{\beta}{ }_{a}=\delta_{\alpha}^{\beta}, E=\operatorname{det}\left(e^{a}{ }_{\alpha}\right)$. Latin indices $a, b, \ldots, m, n, \ldots, s, t, \ldots$ denote a tangent space (a flat index), while Greek indices $\alpha, \beta, \ldots, \mu, v, \ldots \sigma, \tau, \ldots$ denote an Einstein index (a curved index). Letters from the beginning of both the alphabets indicate a general index $(a, b, c, \ldots$ and $\alpha, \beta, \gamma, \ldots)$, from the middle of both the alphabets the observed dimensions $0,1,2,3(m, n, \ldots$ and $\mu, v, \ldots)$, indexes from the bottom of the alphabets indicate the compactified dimensions $(s, t, \ldots$ and $\sigma, \tau, \ldots)$. We assume the signature $\left.\eta^{a b}=\operatorname{diag}\{1,-1,-1, \cdots,-1\}\right) f^{\alpha[a} f^{\beta b]}=f^{\alpha a} f^{\beta b}-f^{\alpha b} f^{\beta a}$.

Let us rewrite the fermion part of the Lagrangean of the action so that it manifests in $d=(3+1)$ the free massless fermion part (first term in Equation (A2)), the interaction of fermions with the vector gauge fields (the second term in Equation (A2)), the interaction of fermions with the scalar fields (the third term in Equation (A2)), and the rest.

$$
\mathcal{L}_{f}=\sum_{m} \bar{\psi} \gamma^{m} p_{m} \psi-\sum_{A, i} \bar{\psi} \gamma^{m} \tau^{A i} A_{m}^{A i} \psi++\sum_{s=7,8} \bar{\psi} \gamma^{s} p_{0 s} \psi+\sum_{t=5,6,9, \ldots, 14} \bar{\psi} \gamma^{t} p_{0 t} \psi,
$$

with $\tau^{A i}=\sum_{s t} c_{s t}{ }^{A i} S^{s t},(s, t)=(5,6, \cdots, 13,14)$, which are generators of the subgroups of $S O(13,1)$, determining charges of fermions, with $A_{m}^{A i}=\sum_{s, t} c^{A i}{ }_{s t} \omega^{s t}{ }_{m}$ [9], $m \in(0,1,2,3)$, which are the corresponding superposition of $\omega_{s t m}([2,4,11]$ and the references therein), describing the known vector gauge fields of the standard model - the colour, weak and hyper gauge fields.

$A_{s}^{A i}=\sum_{s^{\prime}, s^{\prime \prime}} c^{A i}{ }_{s^{\prime} s^{\prime \prime}} \omega^{s^{\prime} s^{\prime \prime}}{ }_{s}$ offer explanation for the scalar higgs and Yukawa couplings of the standard model, with $p_{0 s}=p_{s}-\frac{1}{2} S^{s^{\prime} s^{\prime \prime}} \omega_{s^{\prime} s^{\prime \prime} s}-\frac{1}{2} \tilde{S}^{a b} \tilde{\omega}_{a b s}$, where $s \in(7,8),\left(s^{\prime}, s^{\prime \prime}\right) \in(5,6,7,8),(a, b)$ (appearing in $\left.\tilde{S}^{a b}\right)$ run within $(0,1,2,3)$ and $(5,6,7,8)$.

$A_{t}^{A i}=\sum_{s^{\prime}, t^{\prime}} c^{A i} i_{s^{\prime} t^{\prime}} \omega^{s^{\prime} t^{\prime}}$ t predict new scalar fields, which are involved in the matter antimatter asymmetry in the expanding universe [2] and in proton decay, with $p_{0 t}=p_{t}-\frac{1}{2} S^{t^{\prime} t^{\prime \prime}} \omega_{t^{\prime} t^{\prime \prime} t}-\frac{1}{2} \tilde{S}^{a b} \tilde{\omega}_{a b t}$, $t \in(5,6,9, \ldots, 13,14),\left(t^{\prime}, t^{\prime \prime}\right) \in(5,6,7,8)$ and $\in(9,10, \ldots, 14)$.

The spinor function $\psi$ in Equation (A2) represents all the family members, $2^{\frac{d}{2}-1}=64, d=(13+1)$, (after the break of symmetry caused by the neutrino condensate $[2,4,11]$ ) of all the $2^{\frac{7+1}{2}-1}=8$ families, including fermions and antifermions, Table III. [2]. The rest of families are assumed to have very large 
masses as discussed and proved for a toy model in Ref. [13-16]. The properties of quarks and leptons and antiquarks and antileptons, are presented from the point of view of subgroups of $S O(13,1)$ breaking first into $S O(7,1) \times S U(3) \times U(1)$, keeping connection between handedness and the two $S U(2)_{I, I I}$ charges, and further to- $S U(2)_{R} \times S U(2)_{L} \times S U(2)_{I} \times S U(2)_{I I} \times S U(3) \times U(1)$-representing in $d=(3+1)$ the spin and handedness, the weak charge $\tau^{13}$ of $S U(2)_{I}$, the second $\tau^{23}$ of $S U(2)_{I I}$, the colour charge $\tau^{33}$ and $\tau^{38}$ of $S U(3)$ and $\tau^{4}$ of $U(1)$ for massless quarks and leptons and antiquarks and antileptons, postulated by the standard model.

There are twice separated four families of quarks and leptons in the spin-charge-family theory ([3] and the references therein). (All eight families obtain masses when the scalar gauge fields with the space index $(7,8)$ - third term in Equation (A2)—gain nonzero vacuum expectation values at the electroweak phase transition.)

To the lower four families the observed three families of quarks and leptons contribute [8,17]. By the spin-charge-family theory predicted $S U(2) \times S U(2) \times U(1)$ symmetry of mass matrices, which limits the number of free parameters of mass matrices, the properties of the fourth family could be predicted by fitting free parameters to the experimental data. However, the accuracy of the so far measured $3 \times 3$ mixing (sub)matrices are even for quarks far from the required precision, which would enable prediction of masses of the fourth family members [8]. We predict for the assumed masses of the fourth family of quarks the corresponding matrix elements. Calculations show [8] that the larger the masses of the fourth family-up to $6 \mathrm{TeV}$ seems to be allowed by experiments [18] — the smaller are the unwanted mixing elements which could cause the flavour-changing neutral currents and the better is agreement with the experimental data, which require, due to the observations in Refs. [18], that there should be the fourth family due to the nonunitarity of the $3 \times 3$ so far measured mixing matrix for quarks and that the $4 \times 4$ mixing matrix elements should have the properties: $V_{u_{1} d_{4}}>V_{u_{1} d_{3}}, V_{u_{2} d_{4}}<V_{u_{1} d_{4}}$, and $V_{u_{3} d_{4}}<V_{u_{1} d_{4}}$. Here $u_{i}, d_{i}, i=1,2,3,4$ represent $u, c, t, u_{4}$ and $d, s, b, d_{4}$ quarks. Our results are not in contradictions with the observations.

The lowest of the upper four families is, as evaluated in Ref. [7], the candidate, which can explain (or at least can contribute to) the appearance of the dark matter in the universe. Comparing the results from following the fifth family members in the expanding universe with the astrophysical observations of dark matter and the direct measurements of the dark matter, the predicted masses of the fifth family quarks would be $10^{2} \mathrm{TeV}<m_{q_{5}} c^{2}<4 \cdot 10^{2} \mathrm{TeV}$, and the scattering cross section $\sigma$ for the fifth family neutron at least $10^{-6} \times$ smaller than the cross section for the first family neutron. These values change if the fifth family neutron is not the only source of the dark matter.

The spin-charge-family theory offers several other predictions. The reader can find them in the cited references. The more effort and work is put into this theory the more observed phenomena the theory offers the explanation for.

Starting from a very simple action the theory is limited in degrees of freedom and there is not a lot of space for arbitrariness. It is true from the other side that in the many body problems is difficult to make predictions without the accurate enough experimental data, which suggest the way of breaking symmetries.

\section{References}

1. Mankoč Borštnik, N.S. Spinor and vector representations in four dimensional Grassmann space. J. Math. Phys. 1993, 34, 3731-3745. [CrossRef]

2. Mankoč Borštnik, N.S. Can spin-charge-family theory explain baryon number non conservation? Phys. Rev. D 2015, 91, 0703013. [CrossRef] 
3. Mankoč Borštnik, N.S. Spin-charge-family theory is offering next step in understanding elementary particles and fields and correspondingly universe. In Proceedings of the Conference on Cosmology, Gravitational Waves and Particles, IARD Conferences, Ljubljana, Slovenia, 6-9 June 2016.

4. Mankoč Borštnik, N.S. The explanation for the origin of the higgs scalar and for the Yukawa couplings by the spin-charge-family theory. J. Mod. Phys. 2015, 6, 2244-2274. [CrossRef]

5. Mankoč Borštnik, N.S.; Nielsen, H.B.F. How to generate spinor representations in any dimension in terms of projection operators. J. Math. Phys. 2002, 43, 5782. [CrossRef]

6. Mankoč Borštnik, N.S.; Nielsen, H.B.F. How to generate families of spinors. J. Math. Phys. $2003,44,4817$. [CrossRef]

7. Bregar, G.; Mankoč Borštnik, N.S. Does dark matter consist of baryons of new stable family quarks? Phys. Rev. D 2009, 80, 083534. [CrossRef]

8. Bregar, G.; Mankoč Borštnik, N.S. The new experimental data for the quarks mixing matrix are in better agreement with the spin-charge-family theory predictions. In Proceedings of the 17th Workshop "What Comes beyond the Standard Models", Bled, Slovenia, 20-28 July 2014; pp. 20-45.

9. Mankoč Borštnik, N.S.; Lukman, D. Vector and scalar gauge fields with respect to $d=(3+1)$ in Kaluza-Klein theories and in the spin-charge-family theory. Eur. Phys. J. C 2017, 77, 231.

10. Mankoc Borstnik, N.S.; Nielsen, H.B.F. Why nature made a choice of Clifford and not Grassmann coordinates. In Proceedings of the 20th Workshop "What Comes beyond the Standard Models", Bled, Slovenia, 9-17 July 2017; pp. 89-120.

11. Mankoč Borštnik, N.S.; Nielsen, H.B.F. Understanding the second quantization of fermions in Clifford and in Grassmann space New way of second quantization of fermions-Part I and Part II. In Proceedings of the 22nd Workshop "What Comes beyond the Standard Models", Bled, Slovenia, 6-14 July 2019.

12. Dirac, P.A.M. The Quantum Theory of the Electron. Proc. R. Soc. 1928, Al17, 610.

13. Lukman, D.; Mankoč Borštnik, N.S.; Nielsen, H.B. An effective two dimensionality cases bring a new hope to the Kaluza-Klein-like theories. New J. Phys. 2011, 13, 103027. [CrossRef]

14. Lukman, D.; Mankoč Borštnik, N.S. Spinor states on a curved infinite disc with non-zero spin-connection fields. J. Phys. A Math. Theor. 2012, 45, 465401. [CrossRef]

15. Lukman, D.; Mankoč Borštnik, N.S.; Nielsen, H.B. Families of spinors in $d=(1+5)$ with a zweibein and two kinds of spin connection fields on an almost $S^{2}$. In Proceedings of the 15th Workshop "What Comes beyond the Standard Models", Bled, Slovenia, 9-19 July 2012; pp. 157-166.

16. Mankoč Borštnik, N.S.; Nielsen, H.B. Particular boundary condition ensures that a fermion in $d=1+5$, compactified on a finite disk, manifests in $d=1+3$ as massless spinor with a charge $1 / 2$, mass protected and chirally coupled to the gauge field". Phys. Lett. B 2008, 663, 265-269. [CrossRef]

17. Mankoč Borštnik, N.S.; Nielsen, H.B.F. Do the present experiments exclude the existence of the fourth family members? In Proceedings of the 19th Workshop "What Comes beyond the Standard Models", Bled, Slovenia, 11-19 July 2016; pp. 128-146.

18. Belfatto, B.; Beradze, R.; Berezhiani, Z. The CKM unitarity problem: A trace of new physics at the TeV scale? arXiv 2019, arXiv:1906.02714v1.

(C) 2020 by the authors. Licensee MDPI, Basel, Switzerland. This article is an open access article distributed under the terms and conditions of the Creative Commons Attribution (CC BY) license (http:/ / creativecommons.org/licenses/by/4.0/). 